\title{
Deployment of a Wireless Sensor Network in Mobile Phone Environment
}

\author{
AKPUDUMOGU Oluchukwu Nnanna ${ }^{1}$, SANUSI Muhammad ${ }^{2}$, EBELOGU Christopher Ubaka $^{3}$, \\ ODUWOLE Omolara Oluwakemi ${ }^{4}$
}

\author{
Research Scholar ${ }^{1}$, Lecturer ${ }^{2}$, Research Scholar ${ }^{3-4}$ \\ ${ }^{1-4}$ Department of Computer Science, \\ University of Abuja, Abuja,
}

Nigeria

\begin{abstract}
Wireless mobile sensor network is the collection of autonomous and distributed sensor with additional capacity of mobility. Mobility of sensor nodes adds additional functionality to the wireless sensor network of self-deployment and relocation of sensors. Sensors find their own position and placed themselves over the target area after initial sensor distribution. Different approaches have been proposed for the deployment of mobile sensor by considering different issues. Coverage and Connectivity is the main issue of deployment. Some additional issues have to be considered while deploying mobile sensors like sensor relocation, energy efficient movements of sensors, obstacle adaptability, lifetime of network, fault tolerance etc. This paper basically presents the study of different mobile sensor network deployment approaches with their features and drawbacks with respect to a mobile phone environment. The issues of mobile sensor network deployment are investigated in detail. It further discusses the algorithm and the way of deployment along with the self-deployment. Development in the technology of sensor such as Micro Electro Mechanical Systems (MEMS), wireless communications, embedded systems, distributed processing and wireless sensor applications have contributed a large transformation in Wireless Sensor Network (WSN) recently. It assists and improves work performance both in the field of industry and our daily life. Wireless Sensor Network has been widely used in many areas especially for surveillance and monitoring in agriculture and habitat monitoring. Environment monitoring has become an important field of control and protection, providing real-time system and control communication with the physical world. An intelligent and smart Wireless Sensor Network system can gather and process a large amount of data from the beginning of the monitoring and manage air quality, the conditions of traffic, to weather situations. In this paper, we discuss and review wireless sensor network applications for mobile phone environment. In order to implement a good system, there are several requirements to be followed. From the research, it is also proven that this approach can improve the phone performance, provide a convenient and efficient method and can also fulfill functional requirements.
\end{abstract}

Key Words: WNS (Wireless Sensor Network), Wireless Communications, Mobile Phones.

\section{INTRODUCTION}

A wireless sensor network (WSN) is made up of tens to thousands of interconnected sensors that are randomly or deterministically deployed in a field of interest to monitor various environmental changes such as light, temperature, air pressure. All sensors are connected to a base station (BS) via wireless communication links, and the sensing data are periodically collected and aggregated at the BS. The BS can be connected to a designated Web server that makes it possible for authorized users to remotely access and configure the WSN anytime, anywhere via traditional computers and mobile devices such as iPhone, iPod, iPad, smart phones, etc. Mote Lab, for example, is a Web-based sensor network test bed at Harvard University where a set of permanently deployed sensors is connected to a central Web server which provides a Web interface for data accessing and logging, sensor reprogramming, task creating, and scheduling on the test bed. The unit of a WSN is sensor node. Each sensor node has four major components: (1) sensing transducer, (2) data processor, (3) radio transceiver, and (4) embedded batteries [1].

According to [2], Wireless Sensor Network (WSN) has emerged as an efficient technology for wide variety of applications such as home automation, military application, environmental monitoring, habitat monitoring etc. Use of mobile sensors adds more applications to above list. The wireless mobile sensor network is the collection of autonomous and distributed sensor with 
additional capacity of mobility that can sense or monitor physical or environmental conditions cooperatively. WSNs consist of a large number of small, inexpensive, disposable and distributed autonomous sensor nodes that are generally deployed in vast geographical areas. Sensor nodes are severely constrained in terms of storage, resources, computational capabilities, communication bandwidth and power supply. WSN face many challenges due to these many constraints such as lifespan of network, efficiency and the performance of network. An efficient deployment technique can handle these challenges. Deployment of the sensors over the target field to form the wireless network is an important criterion to be considered in most of the applications. It is a critical issue because it affects cost and detection capability of wireless sensor network .Sensors can be mobile or static. (From Wikipedia, the free encyclopedia).

Motivated by the lack of adequate research in area of object tracking sensor networks, we started our investigation with the basic question: which resource is most required by the sensor node and without it node will not work? We found that all resources are necessary and required by the sensor node but energy is one of the most important resource of sensor node, as power/energy is supplied to the sensor node with the help of batteries which are not easily replaceable.

The research will evaluate the impact of wireless nodes in a mobile network and the wider benefits of its deployment and how these benefits can be improved with its introduction in the phone network environment. Future challenges facing these potential technologies and its impact on the phone environment are also discussed. WSN will play a vital role in making our environment smart in future. It has many useful applications these days and will evolve further with the passage of time.

The proposed protocol is aimed at prolonging the lifetime of the sensor networks by balancing the energy consumption of the nodes. The following steps can be taken to save energy caused by communication in WSN. To schedule the state of the nodes (i.e. transmitting, receiving, idle or sleep).Using efficient routing and data collecting methods. Avoiding the handling of unwanted data as in the case of overhearing.

The following steps aims at saving energy dissipated during communication in WSN

- $\quad$ To schedule the state of the nodes (i.e. transmitting, receiving, idle or sleep).

- Using efficient routing and data collecting methods.

- $\quad$ Avoiding the handling of unwanted data as in the case of overhearing

\section{LITERATURE REVIEW}

Recent advances in semiconductor, networking and material science technologies are driving the ubiquitous deployment of largescale wireless sensor networks (WSNs).Together, these technologies have combined to enable a new generation of WSNs that differ greatly from wireless networks developed and deployed as recently as 5 to 10 years ago. Today's state-of-the-art WSNs have lower deployment and maintenance costs, last longer and are more rugged.

[3] concluded in their study:" A wireless sensor network (WSN) consists of spatially distributed autonomous sensors to monitor physical or environmental conditions (i.e. temperature, sound, vibration, pressure, humidity etc.) and to cooperatively pass their data through the network to a main location. The more modern network is bi-directional, also control of sensor activity. The WSN is built of few to several hundreds or even thousands of sensors of nodes, where each node is connected to one (or sometimes several) sensors."

According to [4], the topology of the WSNs, from a simple star network to an advanced multi-hop wireless mesh network can vary. The propagation technique between the hops of the network can be routing or flooding. A wireless sensor network is made up of three components: Sensors Nodes, Task Manager Node (User) and Interconnect Backbone. Each Sensor Node can contain various sensors and actuators that are used to collect the data and control physical processes. The collected data is transferred to the User through the network that can include Internet segments. Besides collecting the data and controlling actuators, a node may need to perform some computation on the measured data. Direct communication between individual nodes can also be required. The Task Manager Node (User) performs tasks in data storage, analysis and display.

\subsection{WSN Technology Transitions}

Although the technology for large-volume industrial and consumer applications did not exist in the 20th century, both academia and industry recognized the potential for such networks and formed joint efforts to solve the engineering challenges.

Examples of these academic/industrial initiatives include:

- UCLA Wireless Integrated Network Sensors (1993).

- University of California at Berkeley Pico Radio program (1999).

- $\mu$ Adaptive Multi-domain Power Aware Sensors program at MIT (2000). 
- NASA Sensor Webs (2001).

- Zig Bee Alliance (2002).

- Center for Embedded Network Sensing (2002).

The goal of many of these initiatives and standards organizations is to enable high-Volume deployment of WSNs in light industrial and consumer applications by reducing the cost and energy per sensor, while simplifying development and maintenance tasks. Reducing WSN deployment costs while increasing functionality involves major advances in four key technology areas: sensors, CMOS-based semiconductor devices, networking protocols and energy storage/generation technology. The culmination of this effort is the deployment of wireless sensor networks for the emerging Internet of Things (IoT). [5].

\subsubsection{Sensor Types}

The heart of any WSN lies in the sensors. The past decade has seen rapid advancement in multiple sensing technologies:

- Micro-electromechanical systems (MEMS) - gyroscopes, accelerometers, magnetometers, pressure sensors, pyro electric effect sensors, and acoustic sensors

- CMOS-based sensors -temperature, humidity, capacitive proximity, chemical composition

- LED sensors - ambient light sensing, proximity sensing, chemical composition

When combined into a network, these cost-effective sensors enable new applications such as optimizing HVAC control and lighting inside of homes and buildings. According to the US Department of Energy "Annual Energy Outlook 2012" report, HVAC and lighting accounted for 48.1percent of all commercial energy used in the US in 2010, a significant amount of which was wasted due to the absence of smart systems. Most HVAC and lighting systems are programmed on timers at best and do not take into account the physical presence of humans. Using MEMS, CMOS, and LED sensors to track environmental conditions (humidity, temperature, ambient light) and the presence of people (pyro electric, proximity, and acoustic), smart systems can be designed to drastically reduce the overall power used by shutting off power to environmental control when no human presence is detected and dimming light fixtures if the ambient lighting is adequate.

\subsection{Networking Topologies and Protocols}

Wireless sensor networking topologies generally fall into four categories: one-way, bi-directional, star and mesh networks. The first networking protocols were simple one-way communication links, still common in applications such as tire pressure monitoring systems, garage door openers and television remote controls. As the need for more advanced topologies became apparent, networking engineers developed low-memory protocols for bi-directional, star and finally mesh technologies. In addition, the industry is making the transition from proprietary to standardized protocols, similar to the transition in MCUs from proprietary instruction sets and toward 8051-based cores for 8-bit processing and ARM-based solutions for 32-bit applications. [3]

The emergence of cost-effective mesh topologies enables new applications where traditional star topologies come up short. For example, a home lighting application can quickly exceed 30 lights and sensors.

\subsection{Wireless Sensor Architecture}

WSNs consist of tiny and low power sensor nodes that collect data through tiny sensors, process the data and send to particular location. We also describe the types of WSNs with the research work. We include the flaws of existing technology or in a particular type and how we can cover those open holes by using various techniques, protocols or algorithms. 


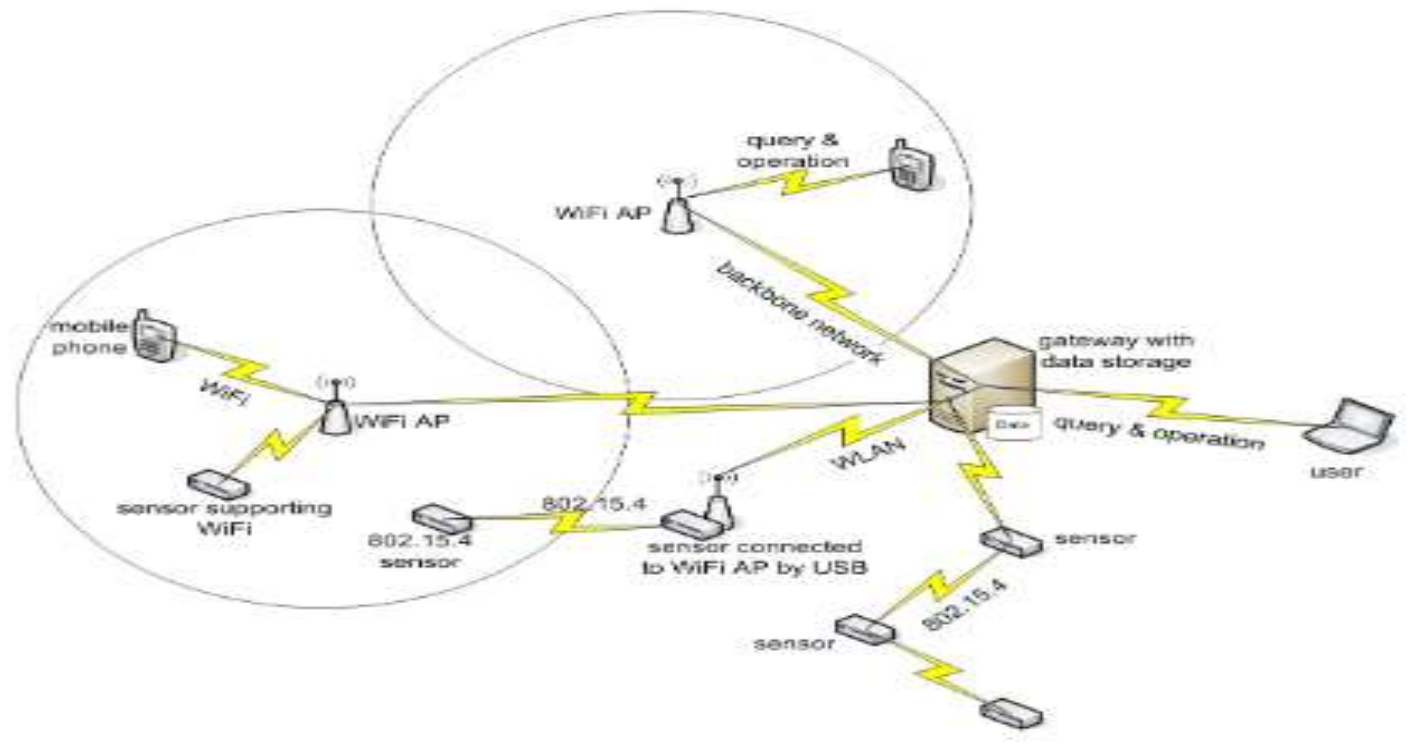

Figure 2.1: Wireless Sensor Architecture

\section{PROPOSED METHODOLOGY}

Our methodology is based on a virtual architecture for the sensor network that enables algorithm design and synthesis. A virtual architecture is an abstract machine model for algorithm design and synthesis and a set of primitives that are independent of low level protocols used to implement them in the underlying network. It is important that the modeling abstractions correspond to mental notions of application behavior on a sensor network system, that formalism exists to specify such behavior, and that some mechanism exists to map the behavior onto the underlying network such that theoretical performance analysis corresponds to real performance measurements.

A key component to the design of any wireless sensor protocol is a thorough knowledge and understanding of the factors that influence the specific network for which the protocol is intended. Therefore a thorough literature study was done to investigate the factors that influence the design of WSN routing protocols. The literature study also includes an investigation into the available WSN routing protocols, in order to identify the common problems faced by these protocols. The new protocol was designed taking into consideration the specific requirement of the application and the common flaws of the available protocols.

A Wireless Sensor Network (WSN) is having a number of sensor nodes connected among them-selves by a wireless medium to perform distributed sensing tasks, which can be used in different types of applications such as surveillance, environmental and health monitoring, and security. Sensor networks are a sensing, computing and communication infrastructure allowing to instrument, observe, and respond to phenomena in the natural environment, and in our day to day life physical and cyber infrastructure. The sensors also can range from small passive micro sensors like "smart dust" to superior range like controllable weather-sensing platforms. Their computation and communication infrastructure is completely different from that found in today's Internet-based systems, which shows the device and application obsessed nature of these systems. An important aspect of WSNs comes from as for the same set of events it has many sensors generating sensing data.

Since number of algorithms and protocols have been proposed for conventional wireless ad hoc networks, which are not well suited to the unique features and application requirements of sensor network. Sensor networks are a new family of wireless networks and are radically different from conventional networks like cellular networks and MANETs. In such conventional networks, the tasks organization, routing and mobility management is performed to optimize the high bandwidth efficiency and Quality of Service (QoS). These networks present superior throughput or delay characteristics under a high level mobility conditions.

\subsection{Problem Formulation}

In this research, the sensing field is represented as a grid of two or three dimensional points $\mathrm{g}_{\mathrm{i}}$. The distance between adjacent grid points is d. It is assumed that sensors can only be deployed in grid points, and the participants' sensing actions are also performed in grid points. The number of grid points in sensing field is denoted by $N$. 


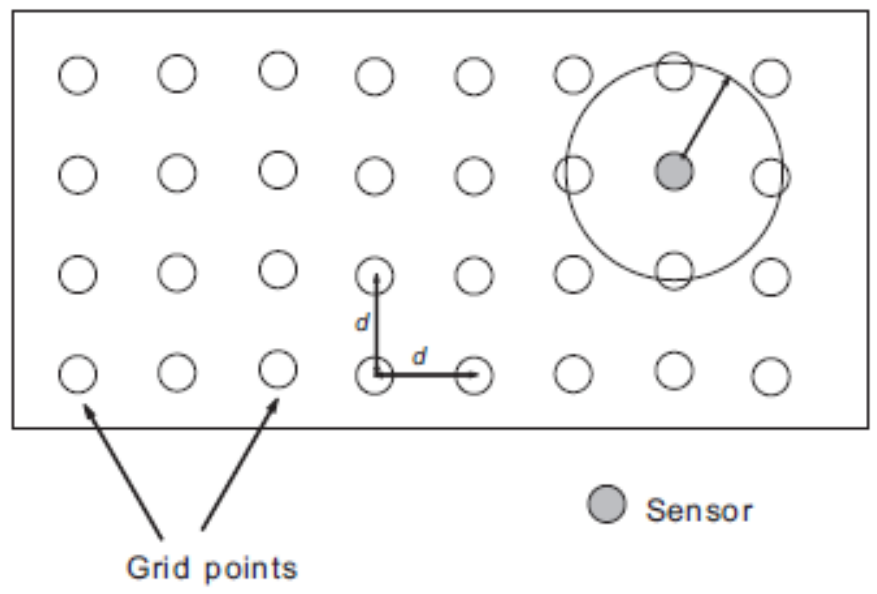

Figure 3.1: Example of grid points in sensing field

Different points in sensing area can have different importance due to different reasons. For example, some points are critical to the sensing project whose data need to be sensed in high priority. And such importance can be changed during the progress of participatory sensing, from period to period. Thus every grid point $g_{i}$ is associated with a pair $\left\langle q_{i}, p_{i}\right\rangle$. $q_{i}$ indicates the lowest quality of data required by the campaign, which is a real number in the range of $[0,1]$. The quality of sensing result is judged by organizes or experts. And $p_{i}$ indicates the lowest coverage probability required for that point. By coverage probability, it means the probability that a grid point $g_{i}$ is sensed by participants or wireless sensors. At the beginning of each period, quality and probability vectors $\mathrm{Q}=\left\langle q_{1}, q_{2} \ldots, q_{N}\right\rangle$ and $p=\left\langle p_{1}, p_{2} \ldots, \mathrm{p}_{N}\right\rangle$ are given as input parameters.

Wireless sensor network should act as a complementary method in participatory sensing, to make sure enough data could be sensed. And it is not wise to deploy the network once and remain it the same during the whole campaign. It should be combined with human actions.

The participatory sensing campaign can be divided into several periods. Before each period, the wireless sensor network is deployed (again), according to the information of participatory sensing campaign and its participants. There are many factors to be considered when a wireless sensor network is deployed, such as energy saving, connectivity, total cost. In most of participatory sensing campaigns, the sensors required are expensive because of their advanced functions. As a result, the most important factor is the number of sensors deployed. The aim is to deploy minimum number of sensors, and provide enough coverage for every grid point in sensing grid at same time.

\section{SYSTEM IMPLEMTATION AND TESTING}

\subsection{Deployment Algorithm}

The proposed framework for deployment of wireless sensor network in participatory sensing environment has a high level of flexibility. It consists of several sub-models, and they communicate with each other by parameters. By flexibility, it means every model can be replaced by another providing the interfaces between models remain the same. This gives the deployment algorithm a good generality, which is important due to diversity of participatory campaigns.

The framework based on the assumption that the sensing field consists of two of three dimensional grid points, as it is described in the problem formulation. The framework concentrates on the two-dimensional cases. However, it can be generalized into three dimensional cases straightforwardly. The distance $d$ between adjacent grid points is determined by different campaigns. The smaller $d$ is, the more precise the frame becomes. However, too small $d$ will result in large number of points in sensing field, which increases the cost for computation. As a result, $d$ should be chosen according to practical situations.

Besides this, the framework consists of following sub-models:

- The model for sensors gives the sensing ability, by providing a detection probability matrix D. Each entry $d_{i, j}$ gives the probability that grid point $g_{i}$ can be sensed by a sensor deployed in grid point $g_{\mathrm{j}}$.

- The terrain model gives information of the sensing field, such as obstacles and climates. It affects the detection probability matrix D. For example, obstacles block the visions of some kinds of sensors and this will set some entries $d_{i, j}$ to be 0 . The climates, such as fog, can decrease the detection probability. As a result, it works with sensors model together to provide the detection probability matrix $\mathrm{D}$. 
- The performance of every participant is described by quality evaluation model. The sensing quality is represented by marks as a real number $q$ in the range of $[0,1]$. This sub-model gives the probability distribution $p(q)$ for sensing quality of every participant, based on historical data. The sensing quality probability of next sensing action in the range $\left[\mathrm{q}_{1}, \mathrm{q}_{2}\right.$ ] can be calculated by $\sum_{q 2}^{q 1} \quad \mathrm{p}(\mathrm{q}) \mathrm{dq}$. For example, if a sensing quality probability distribution of one participant is:

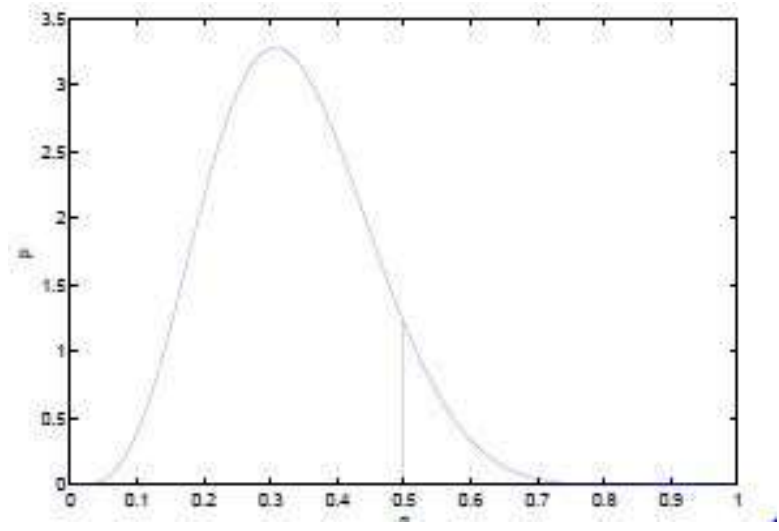

Figure 4.1: Example of sensing quality probability distribution

Then when this participant performs a sensing action next time, the probability that the quality of sensing result lies in the range of $[0.5,1]$ is given by $\sum_{0.5}^{1} \quad p(q) d q$.

- The probability that a participant performs a sensing action in grid point $g_{i}$ in next period is predicted by the participant's actions predication model. This model provides a vector $V$, in which $V_{i}$ gives the probability that grid point $g_{i}$ will be visited next period.

- The deployment algorithm takes the input from above sub-models, then calculates the grid points which need to be monitored by extra sensor(s) and gives the minimum wireless sensors required to make sure every grid point is covered by its minimum sensing probability, as well as the locations where the sensors are deployed.

The whole framework can be shown in figure 4.2 :

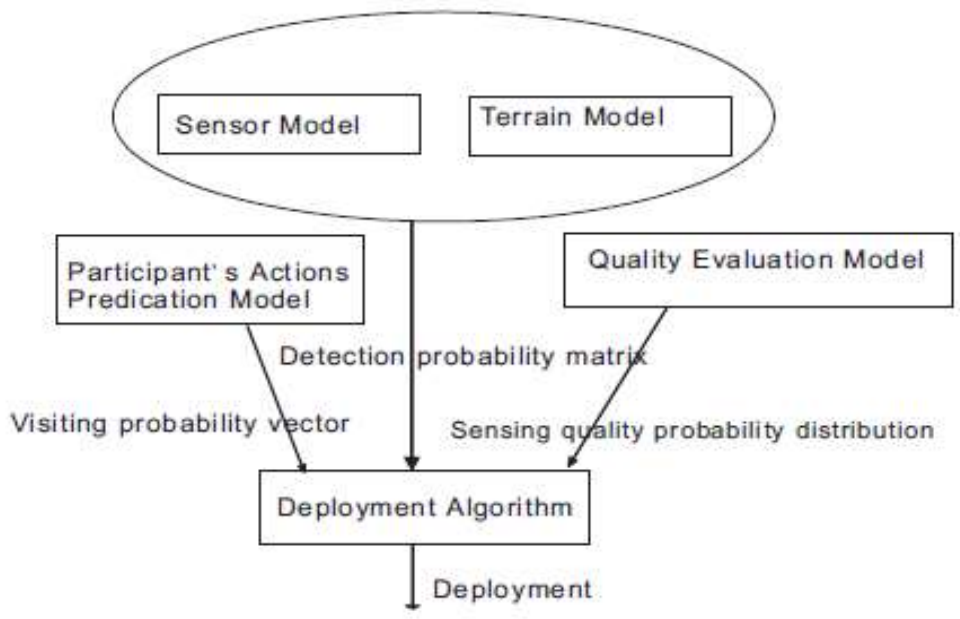

Figure 4.2: Framework for the model 


\subsection{Model for Sensors And Terrain Of Sensing Field}

In a participatory sensing campaign, there are two kinds of sensors: mobile phones and wireless sensors. We represent sensors as a disk centered in one point with a positive detection radius $r$, which represent the range of sensing. Because participants can have different kinds of mobile phones, their sensing ranges differ from each other. Generally speaking, there are two kinds of sensing precise and imprecise, which correspond two different cases in the algorithm:

- Case 1: The sensors can produce "perfect" detections. That means, the result produced by the sensors is either "yes" or "no", as a binary result. For example, in harbor communities study, participants are asked to gather data of gaseous and particulate pollutants. In the sensing range of a sensor, the data won't differ much, thus it can be regarded as "precise".

- Case 2: The detections of sensors are imprecise. The precision of data is affected by some factors, such as target distance to the sensor. For example, in the cycle sense campaign, participants are asked to gather acoustic data which are used to analyze the noises near the cycle route. The sound becomes weaker as the target distance increases. As a result, the detection probability decreases.

Singh Dhillon in 2003 proposed a model for probability of detection of a target by a sensor, together with a model for terrain of sensing area. The assumption is that the detection probability varies exponentially with the distance between the target and the sensor. A target at distance $d$ from sensor is detected by that sensor with probability:

$$
p(d)= \begin{cases}e^{-\alpha d} & \text { if } 0 \leq d \leq r \\ 0 & \text { otherwise }\end{cases}
$$

However, the choice of a sensor detection model is a parameter to the algorithms. It can be changed without affecting the algorithms. It can be noted that case 1 is a specialization of case 2 , by setting

$$
p(d)= \begin{cases}1 & \text { if } 0 \leq d \leq r \\ 0 & \text { otherwise }\end{cases}
$$

Terrain is an important factor in wireless sensor networks, which heavily affects the range of sensors. For example, obstacles such as buildings can block the vision of sensors. We represent the sensing field of interested as a grid (two- dimensional) of points. However, the proposed algorithm can be generalized into three- dimensional cases straightforwardly. The following figure shows an example of sensing field with obstacles:

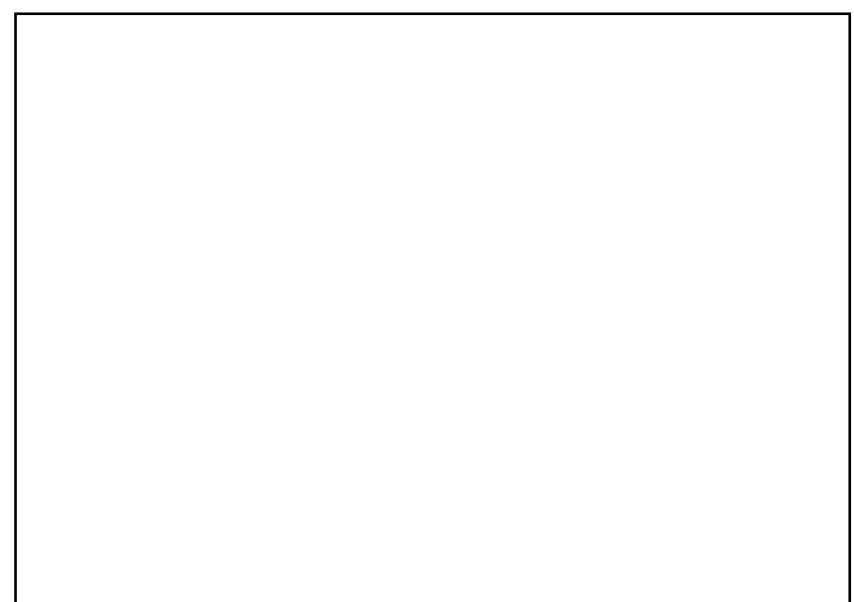

Figure 4.3: Example of grid points, obstacles and a sensor

Detection probability matrix D describes the probability of detection be-tween sensors and targets:

$$
D=\left(\begin{array}{cccc}
d_{1,1} & d_{1,2} & \cdots & d_{1, n} \\
d_{2,1} & d_{2,2} & \cdots & d_{2, n} \\
\vdots & \vdots & \ddots & \vdots \\
d_{n, 1} & d_{n, 2} & \cdots & d_{n, n}
\end{array}\right)
$$


In which $d_{i, j}$ indicates the sensing probability of a target in grid point $j$ by a sensor in grid point $i$. The probability matrix can be calculated according to knowledge of sensor and terrain models. We let $d i s(i, j)$ denote the distance from grid point $i$ to grid point $j$. Then in our thesis, entries of $\mathrm{D}$ are calculated as follows:

$$
d_{i, j}= \begin{cases}p(\operatorname{dis}(i, j)) & \text { if vision of grid point } j \text { from } i \text { cannot be blocked } \\ 0 & \text { otherwise }\end{cases}
$$

\subsection{Evaluation of Performance of Participants}

Evaluating performance of participants is similar to evaluating reputation of transaction parties in e-commerce. The participants act as merchants who sell goods, and the organizers or experts act as the customers. In reputation systems for e-commerce, the reputations of merchants are calculated according to the feedbacks and remarks from customers. Thus in participatory sensing, the organizers can also use similar systems to evaluating performance of participants. Existing reputation systems used in applications include: cumulative, where a user's reputation ratings are summed; average, where the reputation score is computed by averaging all scores; blurred, where a weighted sum is used to down weight old ratings; and adaptive, where the current reputation score affects to what degree new observations make a difference.

\subsection{Determination of grid points which need extra wireless sensors}

For every grid point $g_{i}$ which has the sensing requirement $\left\langle q_{i}, p_{i}\right\rangle$, the probability that its data can be sensed by any participant with required quality can be calculated in following steps:

The probability that participant $x$ perform a sensing action with required quality is

$$
\int_{q \mathrm{x}}^{1} f_{x}(q \mid \alpha, \beta) \mathrm{d} q
$$

The probability that grid point gi can be sensed when action is performed in grid point $j$ is

$$
d_{j, i}
$$

As a result, the probability that grid point $i$ data can be sensed by participant $x$ with required quality is.

$$
1-\prod_{j=1}^{j=N}\left(1-\left(d_{j, i} \int_{q,}^{1} f_{x}(q \mid \alpha, \beta) \mathrm{d} q\left(1-\prod_{t=0}^{\infty}\left(1-P_{0, j}^{\prime(t)}\right)\right)\right)\right)
$$

Now to can get the probability that data grid point $i$ can be sensed by any participant with required quality is

$$
p r_{i}=1-\prod_{x \in \text { paritipants }} \prod_{j=1}^{j=N}\left(1-\left(d_{j, i} \int_{q_{i}}^{1} f_{x}(q \mid \alpha, \beta) \mathrm{d} q\left(1-\prod_{t=0}^{\infty}\left(1-P_{0, j}^{\prime}(t)\right)\right)\right)\right.
$$

If the result is smaller than $p_{i}$, it means grid point $i$ need be monitored by extra wireless sensors. And it's not hard to get that the new lowest required probability that grid point $i$ is monitored by wireless sensors is

$$
\begin{array}{ll}
p_{i}^{\prime} & = \begin{cases}\frac{p_{i}-p r_{i}}{1-p r_{i}} & \text { if } p r_{i} \leq p_{i} \\
0 & \text { otherwise }\end{cases}
\end{array}
$$

After this step, we get a new vector $P^{\prime}$ which indicates the probability that a grid point $\mathrm{g}_{\mathrm{i}}$ need to be covered by extra wireless sensors. 
International Journal of Advances in Scientific Research and Engineering (ijasre), Volume 6 (7)，July -2020

\subsection{Deployment of wireless sensor network}

Different algorithms were given for the two different cases. Unfortunately, both cases are NP-hard. Firstly, we propose a constraint programming algorithm to solve case 1, which aim at finding the optimal solution. Secondly, a greedy heuristic algorithm is proposed for case 2 , which can find an approximate solution. The performances of these algorithms are studied and compared in several different study cases.

\subsubsection{Solve case 1 by constraint programming}

\section{Minimum set covering}

The set covering problems (SC) can be defined as follows: let $U=\left\{\mathrm{e}_{1}, e_{2}, \ldots, \mathrm{e}_{\mathrm{m}}\right\}$ be a set of $m$ elements. Let $\mathrm{X}$ be as collection of subset of $\mathrm{U}$, i.e. $\mathrm{X}=\left\{S_{1} \ldots S_{n}\right\}$ where $S_{i} \leq U,(1 \leq i \leq n)$ and let $c_{j}$ be a weight associated to each subset $S_{j, 1} \leq j \leq n$. SC calls for a subset $T$ of indexes of $X$ covering $U: T \leq\{1, . . n\}^{\wedge} U_{i} E T S_{i}=U$ and such that $\sum_{\mathrm{j} E T c j} \quad$. This research only considered

$c_{j}=1$.

Case 1 can be modeled as minimum set covering problem as follows: Let $U$ be the set of grid points which need to be monitored by extra wireless sensor. For each grid point $g_{i}$ in $U$, a subset $S_{i}$ of $U$ is built by adding all the grid points $g_{i}$ such that $d_{i, \mathrm{j}}=1$.

There are several techniques could be used to solved the minimum set covering problem.

For example, constraint programming, integer programming and local search. We choose constraint programming to solve case 1 in this research.

\section{Preliminaries}

The minimum set covering problem can be viewed more straightforwardly as a bipartite graph

$\langle A, B, E\rangle$, by constructing vertex set $A$ in which $a_{i}$ represents $S_{i}$, and vertex set $B$ in which $b_{j}$ represents $e_{j}$, and connecting vertex $a_{i}$ with $b_{j}$ if $e_{j} \in S_{i}$. Then the minimum set covering is converted to finding smallest subset $A^{I} € A$ to "cover" all vertexes in $B$. By cover" it means every vertex in $B$ has at least one neighbor in $\mathrm{A}^{\mathrm{I}}$.

For example: $U=\{1,2,3,4,5\}, S_{1}=\{1,3,5\}, S_{2}=\{1,4\}, S_{3}=\{5,2\}, S_{4}=\{1,3,2\}$. It could be viewed as following bipartite graph.

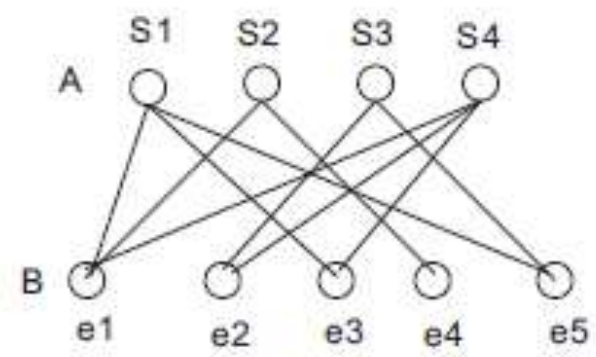

Figure 4.4: View as bipartite graph

One of the smallest $\mathrm{A}^{\mathrm{I}}$ is $\{\mathrm{a} 1, \mathrm{a} 2, \mathrm{a} 4\}$.

Let $m c s(S, U)$ denote the cardinality of smallest set cover of $S$ with the universe $U$. Meanwhile, let $m s c G(\mathrm{~A}, \mathrm{~B}, \mathrm{E})$ denote the cardinality of smallest subset of $A$ which covers all vertexes in $B$, with the edges set $E$. And can also use $m s c G(G)$ if the detailed information about graph $\mathrm{G}$ is not cared.

Let $S$ be a subset of $U$. By $\operatorname{del}(\mathrm{X}, \mathrm{S})$ we denote the collection which is obtained from $X$ by removing the elements of $S$ from every $S_{i}$ in $X: \operatorname{del}(X, S)=\left\{S^{I} \mid S^{I}=S_{i} \backslash S^{\wedge} S_{i} \in X\right\}$. 
International Journal of Advances in Scientific Research and Engineering (ijasre), Volume 6 (7)，July -2020

\section{Algorithm for the proposed framework}

The proposed algorithm for searching for a minimum set covering. MinimumSetCovering will find the minimum set covering in bipartite graph (A, B, E). Current is the vertex set which has been chosen as part of set covering. $K$ is the minimum set covering found so far. FilterAndPropagate will do the propagation, it returns false if this search branch fails. At the beginning, it is called by $C P(A, B, E)$.

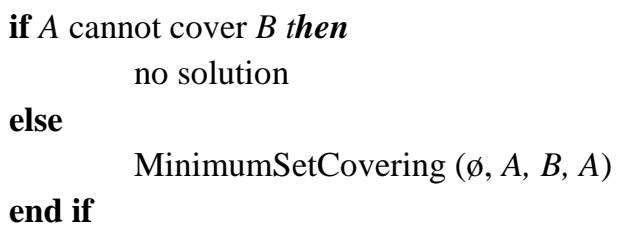

MinimumSetCovering works as follows: first it will try to select vertex $a_{i}$ from A by some strategy, until a covering set is found or the search fails. After selecting $a_{i}$, it will remove all $b_{j}$ in $B$ which is covered by $a_{i}$. Then it will call FilterAndPropagate to perform propagations. If FilterAndPropagate returns false, it increase the fails counter. Otherwise, it checks whether B is empty, if so, Current will be a set covering and $\mathrm{K}$ will be replaced if $|\mathrm{K}|>\mid$ Current $\mid$. Otherwise it calls MinimumSetCovering to recursive. MinimumSetCovering (Current, A,B,K).

while $A$ is not empty do save $B$

select $a$ from $A$ by some strategy and remove it as well as the covered elements in $B$

save A

add $a$ to Current

if FilterAndPropagate (Current, $A, B, C$ ) then

if $B$ is empty then

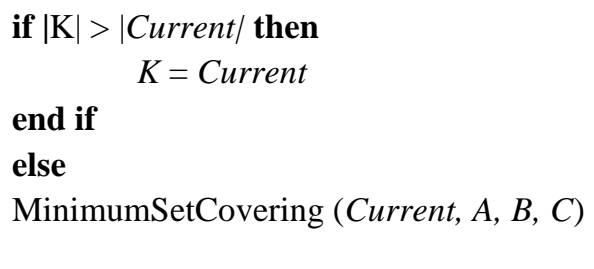

else

$$
\text { fails }=\text { fails }+1
$$

end if

remove $a$ from Current

restore $A$

restore $B$

end while

FilterAndPropagate does the propagation

FilterAndPropagate (Current, $A, B, C$ )

repeat

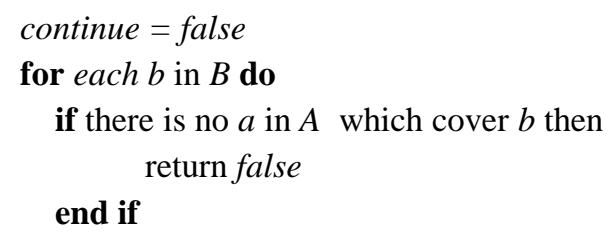

end for

for each $a$ in $A$ do

if the elements covered by $a$ is subset of another $a$ then

remove $a$ from $A$

continue $=$ true

endif

end for

for each $b$ in $B$ do

if there is only $a$ in $A$ which can cover $b$ then

remove $a$ from $A$ and all the $b$ in $B$ which is covered by $a$ 
International Journal of Advances in Scientific Research and Engineering (ijasre), Volume 6 (7)，July -2020

add $a$ to current

continue $=$ true

end if

end for

until continue is false

There are several possible strategy could be used to select the next $A_{i}$ to enlarge current

1. One possible strategy is to select $a_{i}$ which covers the most $b_{j}$.

2. Another way is first looking for $b_{j}$ which is covered by fewest $a_{i}$, then choose one $a_{i}$ which covers it.

3. If after selecting some $a_{i}$, the graph has more than one connected components, then we get several sub problems with smaller scales. Thus we could solve them individually.

\subsubsection{Solve case 2 by greedy algorithm}

Case 2 is also NP-hard, simply because it is a generalization of case 1. Firstly we propose an algorithm based on integer linear programming, and then a polynomial greedy heuristic algorithm is proposed to solve it.

Integer linear programming formalization Case 2 can be formalized by integer linear programming, as follows: Let ai be a binary variable defined as follows:

$$
a_{i}= \begin{cases}1 & \text { if a wireless sensor is deployed in grid point } g_{i} \\ 0 & \text { otherwise }\end{cases}
$$

Then for grid point $g_{i}$, the probability that it cannot be monitored by any wireless sensors is:

$$
\prod_{j=1}^{j=N}\left(1-a_{j} d_{j, i}\right)
$$

Thus case 2 can be formalized as:

objective: Minimize $\sum a_{i}$

subject to:

$$
\begin{aligned}
& \text { for every grid point } i, \prod\left(1-a_{j} d_{j, i}\right) \leq 1-p_{i} \\
& \qquad 0 \leq a_{i} \leq 1
\end{aligned}
$$

However, this formalization is not in an integer linear programming form, because some of its constrains are not linear. With help of some tricks, it can be converted into linear form: First, with help of logarithmic function, the constrains can be converted into:

$$
\sum l g\left(1-a_{j} d_{j, i}\right) \leq l g\left(1-p_{i}\right)
$$

The only problem is that logarithmic function $f(x)=\lg (x)$ is undefined for $\mathrm{x}=0$. But this can be solved by adding a negligibly small value to each item. For example:

$$
\sum \lg \left(1+10^{-100}-a_{j} d_{j, i}\right) \leq \lg \left(1+10^{-100}-p_{i}\right)
$$

Then the integer variables are moved outside the logarithmic functions:

$$
\sum a_{j} \lg \left(1+10^{-100}-d_{j, i}\right) \leq \lg \left(1+10^{-100}-p_{i}\right)
$$

Now the constraints become linear. As a result, case 2 can be formalized as: 
objective: Minimize $\sum a_{i}$

subject to:

$$
\begin{gathered}
\sum a_{j} l g\left(1+10^{-100}-d_{j, i}\right) \leq \lg \left(1+10^{-100}-p_{i}\right) \\
0 \leq a_{i} \leq 1
\end{gathered}
$$

for every grid point $i$

Although case 2 can be formalized into an integer linear programming form, it cannot be solved efficiently when the scale of problem increases. As a result, a greedy heuristic algorithm is been proposed here.

\section{Greedy algorithms}

The proposed greedy algorithm method is called MAX_V ALID_COV and the pseudocode steps

are outlined as follows:

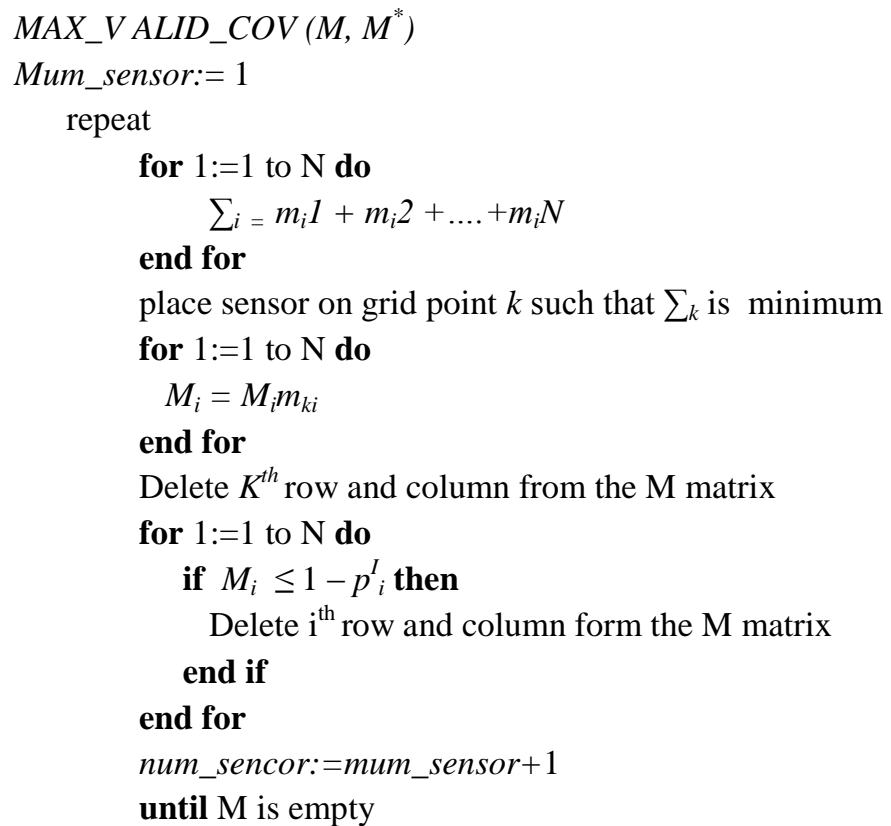

Performance of MAX VALID COV; When the scale of problem increases, the constraint programming algorithm takes more and more time to find the optimal solution. However, the greedy algorithm has a time complexity of $O\left(N^{2}\right)$, which is suitable for large scale of deployment. As a result, the greedy algorithm can be used to get an approximate solution for case 1. In this, greedy algorithm is compared with constraint programming algorithm. The results show that our greedy algorithm is a good approximation of the optimal solutions.

\section{SUMMARY AND FUTURE WORK}

We propose a framework for wireless sensor network deployment in mobile phone assisted environment. We suggest that wireless sensors and mobile phone participants can perform sensing collaboratively and complement each other. Our framework predicts the sensing quality of the mobile phone participants considering their mobility and sensing behaviors.

Then, it provides wireless sensor deployment minimizing the number of sensors, while guaranteeing satisfactory sensing quality and coverage. Our framework includes several sub-models which offer high level of flexibility. It can adapt to different kinds of sensing campaigns by replacing any of the sub-models accordingly. Extensive evaluations with real mobile traces have shown that our framework can provide good coverage and sensing quality in most of the grid points with small number of additional wireless sensors. We believe that the performance of our framework will improve further if we understand the behavior and motion patterns of the participants thoroughly in real campaigns 
The experiments show that our framework has a good performance. It covers most of the grid points with required sensing probability. And the performance will become even better in real campaigns. Because the participants' behaviors have some hidden patterns and contain a lot of information which can be learned by our framework.

\section{CONCLUSION}

We propose a framework for wireless sensor network deployment in mobile phone assisted environment. We suggest that wireless sensors and mobile phone participants can perform sensing collaboratively and complement each other. Our framework predicts the sensing quality of the mobile phone participants considering their mobility and sensing behaviors. Then, it provides wireless sensor deployment minimizing the number of sensors, while guaranteeing satisfactory sensing quality and coverage. Our framework includes several sub-models which offer high level of flexibility. It can adapt to different kinds of sensing campaigns by replacing any of the sub-models accordingly. Extensive evaluations with real mobile traces have shown that our framework can provide good coverage and sensing quality in most of the grid points with small number of additional wireless sensors. We believe that the performance of our framework will improve further if we understand the behavior and motion patterns of the participants thoroughly in real campaigns.

Wireless sensor networks (WSN) are used in many applications that range from smart home, to telemedicine, to transportation, to space explorations. Because of the local processing capabilities added to the sensors a lot of decisions can be made locally reducing the communication time between the sensor/actuator and the decision making entity. Because of this improved feedback time and improved intelligence associated with modern sensor devices, wireless sensor network are proliferating in control systems and space exploration systems. Given that there are networking technologies that can be used with some deterministic delay bounds, along with sensors that utilize miniaturized technologies; the weight factor is significantly reduced along with improved reliability. They will further find applications in control systems with stringent requirements. These advantages bring certain challenges that need to be addressed, including

- Wireless channel is prone to interference and therefore the number of devices that can be connected should be designed to satisfy the signal to noise ratio requirements.

- Reliability: studies need to be conducted on fault tolerance and QoS reliability in space environments.

- Optimum placement of sensor transceivers within the space habitat for consistent radio link connectivity.

In order to make the mobile application more efficient and perceptible to the user, we intend to improve it by providing feedback about the user navigation on the application. Similarly, we also intend to implement the zoom feature to enable enlarging or reducing the size of the table with the historic of data, so that people who want to browse data in a larger size may do so. We also intend to give the user the possibility of sorting historic data by a parameter chosen by the user, i.e., to allow the user to sort data by date, rooms, floors, or sensors, showing data in a descending or ascending order. It is desirable that the module generating a gradient map of the environmental parameters is implemented, which will allow displaying data from a particular sensor in a more intuitive manner. This makes it possible to give a more specific idea of what is occurring, allowing the user even to check if a particular sensor needs to be moved to another location or replaced by another one.

The tests of this application were based on data collected from a small test bed installed in the laboratory. The WSN was not installed in the museum yet since the equipment for assembling the monitoring devices did not arrive in time. Thus, the application was tested with some data to permit to observe its basic operation.

Wireless sensor networks hold great promises in providing a new networking paradigm which allows for interacting with the physical world. Though each individual sensor may have severe resource constraints in terms of energy, memory, communication and computer capabilities, thousands or even hundreds of thousands of them may collectively monitor the physical world, disseminate information upon critical environmental events, and process the information on a fly. They enable potential numerous, new application such as forest fire monitoring, chemical and biological agent detection, soil pollution measurement and autonomous ocean ecology sampling. Toward this end, efficient data dissemination protocol serves as a critical component to realize a large scale sensor network. 
International Journal of Advances in Scientific Research and Engineering (ijasre), Volume 6 (7)，July -2020

\section{REFERENCES}

[1] Fischione C, 2014, "Principles of Wireless Sensor Networks - Lecture 2, Introduction to Programming WSNs" KTH Royal Institute of technology, Stockholm, Sweden.

[2] Carlos-Mancilla M, López-Mellado E and Siller M, 2016, "Wireless Sensor Networks Formation: Approaches and Techniques". Review Article - Open Access. Retrieved from: https://www.hindawi.com/journals/js/2016/2081902/

[3] Bokare M and Ralegaonkar A, 2012, "Wireless Sensor Network: A Promising Approach for Distributed Sensing Tasks". Excel Journal of Engineering Technology and Management Science, Vol. 1 No.1, pg 1-9. December-January 2012.

[4] Dargie, W. and Poellabauer, C. (2010). Fundamentals of wireless sensor networks: theory and practice. John Wiley and Sons. pp. 168-183, 191-192. ISBN 978-0-470-99765-9.

[5] J. Zhang, L. Shan, H. Hu and Y. Yang, "Mobile cellular networks and wireless sensor networks: toward convergence," in IEEE Communications Magazine, vol. 50, no. 3, pp. 164-169, March 2012, doi: 10.1109/MCOM.2012.6163597. 